\title{
Emergency admissions for assessment at All Saints Hospital
}

\author{
David J. Fox, Senior House Officer in Psychiatry, Mid-Trent Rotation, \\ Peter Hodgkinson Centre, County Hospital, Greetwell Road, Lincoln LN2 5QY
}

During 1991 , only $0.54 \%$ of all admissions to All Saints Hospital (ASH) were under section 4 of the 1983 Mental Health Act (MHA) (England \& Wales), representing $2.63 \%$ of all admissions under the MHA. These figures compare favourably with other published studies. This paper looks at possible reasons for these low figures and reports on an audit of those few admissions against the criteria laid down in the MHA.

Section 4 of the Mental Health Act allows for admission to hospital for 72 hours assessment in an emergency on the recommendation of one doctor and an approved social worker (ASW). Its use is recommended only when a second doctor is unavailable to support an application for admission under section 2 or 3 . Criteria for an emergency are the significant risk of harm to the patient, others or property and/or the need for physical restraint of the patient (DOH, 1990). Published studies (Winterson \& Barraclough, 1984, 1985; Webster et al, 1987; Puri \& Beringham, 1990) show the use of section 4 varying between $0.8-1.6 \%$ of all admissions and $7.54-30 \%$ of admissions under the MHA. The use of section 4 of the MHA at ASH was audited and the number of admissions was found to be surprisingly small. Possible reasons for this will be discussed later.

\section{The study}

ASH is an inner city Victorian psychiatric hospital with a catchment population of 425,000 , with a large ethnic subgroup.

Statistics on all admissions from 1 January to 31 December 1991 (inclusive) were collated and all patients admitted under section 4 were identified and their notes reviewed. Specifically, the section papers were scrutinised to assess how closely the clinical circumstances adbered to the criteria laid down in the MHA. Areas for improvement were identified.

\section{Findings}

Of 1495 admissions, only eight $(0.54 \%)$ were admitted under section 4 . This corresponds to $2.63 \%$ of the 304 patients admitted under the MHA to ASH in 1991. Of these eight, one set of notes was lost and a further three sets did not contain copies of the medical or social work recommendations for admission under section, so copies of the originals were taken from files kept by the statistics department.

For three of the cases, the social worker concerned gave no reason for the unavailability of a second opinion doctor, although the patient may have been known to the recommending doctor in these cases. Of the reasons given, one patient had recently moved catchment areas and his previous GP had retired; one was "extremely elusive" and organising two doctors, an ASW and the patient together had proved impossible; another had come from Manchester that day and was not known to any local doctor; in one case the ASW had tried to contact six second opinion doctors but all were unavailable; in another the responsible medical officer was at a conference and the GP was unobtainable. The last case was the only time a hopital doctor could not be contacted.

Although it is mandatory for the medical practitioner to complete an entry specifying reasons why the patient should be admitted urgently without a second opinion, this was not done in one case, but an extensive entry was made by the ASW. However, this should not have been accepted by the hospital managers. For the others, details of appropriate reasons for urgent admission were given.

Following admission, second opinions were obtained for six of the patients and they were converted to observation or treatment orders within 24 hours. One patient repeatedly absconded and so a second opinion was not possible and the patient, who lived over 60 miles away, discharged himself when his section lapsed. No details are available on the outcome of the eighth patient, whose notes were missing.

\section{Comment}

The small (0.54) percentage of admissions under the emergency order compares well with all other published studies, which report rates of $0.8-1.6 \%$, and our figure of $2.63 \%$ of all sections is many times lower than any other study (7.54-30\% Winterson \& Barraclough, 1984, 1985; Webster et al, 1987; Puri \& Bermingham, 1990). This can readily be explained by 
the nature of our catchment area. ASH is an inner city psychiatric hospital and as a consequence has a substantial population of chronically ill patients who, typically, relapse with limited insight and require enforced treatment or supervision in hospital. The other published figures are for wider catchment areas, thus diluting this effect. The hospital's policy of community treatment can also mean that when patients do get admitted their illness is severe with, again, those typical features, requiring detention. The proportion of admissions to ASH under the MHA is over $20 \%$ whereas Winterson \& Barraclough (1985) and Webster et al (1987) report between 11.8 and $14.52 \%$ of compulsory admissions. Therefore, our low rate of section 4 admissions as a percentage of compulsory detentions is not entirely surprising, assuming that the total includes a disproportionate number of detained patients. It may also reflect contact, by ASWs, with a greater number of second opinion doctors, because of frequent use of the MHA, and admission avoided.

It is interesting to compare use of section 4 of the MHA with its equivalent, section 24, of the Scottish Mental Health Act of 1984. This allows for detention for 72 hours in an emergency on the recommendation of one registered doctor and a relative or ASW where practicable. In some instances patients could be detained for three days solely on the recommendation of their general practitioner. Section 24 is the commonest method of formal admission for patients in Scotland (Humphries, personal communication), in complete contrast to the 'ideal' of the MHA in England and Wales, to which this audit was directed.

Despite the low numbers of section 4 admissions to ASH, there is still room for improvement. Although the reasons given for the emergency detention of the patients in this study are all acceptable the reasons for a second opinion doctor being unavailable are not, particularly when, within 24 hours, a second opinion was obtained for three-quarters of them. In particular, the case in which "six second opinion doctors were tried and none were available" is unsatisfactory. Emergency access to a medical second opinion, either GP or section 12 approved, needs to be easier than it would appear to have been in this instance.

Furthermore, giving no reason for the unavailability of a second doctor is not acceptable. This is perhaps a fault of the design of the form itself, allowing a medical recommendation or that this part of the form was overlooked.
The single form without a statement from the recommending doctor about the circumstances requiring urgent admission is most likely an oversight, but illustrates that care should be taken in completing Section papers.

From an administrative viewpoint, a copy of the section papers should be kept in the notes of each patient detained under a section of the MHA and not be removed even after discharge. This allows access to what may be the only available information on the circumstances leading to urgent admission. Usually, patients are brought to the hospital by the Police, who are not aware of the circumstances leading to detention, patients seldom know why they are detained because of limited insight (assuming they will talk at all) and no notes accompany patients. Unless events leading to admission are documented at the time, they may never be recorded at all and this was found to be the situation for those notes where the section papers are missing. It would also be helpful if either the sectioning doctor or ASW, or at least some written details, could accompany the patient to hospital.

In summary, ASH received a low proportion of patients under section 4 of the MHA during 1991. The figure could be lower still if a second opinion doctor was more readily available. ASWs should comment in the appropriate space on the recommendation form that the patient is known to the recommending doctor; if not, a reason for the unavailability of a second opinion doctor should be given. Care should be taken to ensure that the forms are completed fully and a duplicate copy of all paperwork should be filed in the patients' notes. Some details should accompany the patient to hospital, if not the doctor or ASW in person.

\section{References}

Department of Health (1990) Code of Practice, the 1983 Mental Health Act. HMSO. Pp. 72-73.

Puri, B. K. \& Bermingham, D. F. (1990) High rate of Section 4 admissions: clinical implications and possible explanation. Psychiatric Bulletin, 14, 21-22.

Webster, L., Dean, C. \& Kessel, N. (1987) Effect of the 1983 Mental Health Act on the management of psychiatric patients. British Medical Journal, 295, 1529-1532.

Winterson, M. J. \& Barraclough, B. M. (1984) Effect of 1983 Mental Health legislation on compulsory admissions to a district general hospital. Lancet, if, 44. _ \& - (1985) Effects of 1983 Mental Health Act. Lancet, ii, 1426. 